\title{
Commentary: Decision making in thoracic aortic surgery: One size fits all?
}

\author{
Florian S. Schoenhoff, MD, and Thierry Carrel, MD
}

\footnotetext{
From the Department of Cardiovascular Surgery, University Hospital Bern and University of Bern, Bern, Switzerland.

Disclosures: Authors have nothing to disclose with regard to commercial support.

Received for publication Sept 20, 2018; accepted for publication Sept 21, 2018; available ahead of print Nov 3, 2018.

Address for reprints: Thierry Carrel, MD, Department of Cardiovascular Surgery, University Hospital and University of Bern Freiburgstrasse, CH-3010 Bern, Switzerland (E-mail: thierry.carrel@insel.ch).

J Thorac Cardiovasc Surg 2019;157:1748-9

$0022-5223 / \$ 36.00$

Copyright (c) 2018 by The American Association for Thoracic Surgery

https://doi.org/10.1016/j.jtcvs.2018.09.104
}

In the early 20th century, decades before replacement of the thoracic aorta became feasible, Sir William Osler ${ }^{1}$ already noted "no disease is more conducive to clinical humility than aneurysm of the aorta."

Although replacement of the aortic root and ascending aorta can now be performed with low mortality, the question of when to operate on the individual patient is still ongoing. There has never been a prospective trial randomizing patients into those undergoing surgery or not at any given diameter. Given the fact that the diameter of the aorta cannot be reliably measured once dissection occurred, there is relative paucity of reliable data to support the decisionmaking process.

Over time, we have learned that rather large aneurysms have a high risk of dissection or rupture and therefore exceed the risk of elective surgery in the majority of patients. But in patients presenting with an aneurysm of the ascending aorta between 4 and $5 \mathrm{~cm}$, the situation remains unclear.

In this edition of the Journal, Saeyeldin and colleagues, ${ }^{2}$ from Yale, New Haven, report data using a simplified approach for decision making. The group recommended surgery to patients with an aortic diameter greater than $5 \mathrm{~cm}$. Patients with an aortic diameter between 4 and $5 \mathrm{~cm}$ received medical treatment and regular follow-up. If patients in the latter group had a strong family history of dissection, connective tissue disorder, bicuspid aortic valve, or chest pain, surgery was recommended as well.

The study is remarkable in several ways: It combines expert surgical treatment with large patient numbers and an excellent follow-up over a long period of time, all while demonstrating a high adherence to the proposed treatment algorithm.

The study demonstrated a remarkably high rate of aortic events in those patients who presented with an aortic diameter greater than $5 \mathrm{~cm}$ but did not undergo surgery because of comorbidities or because surgery was refused. In this

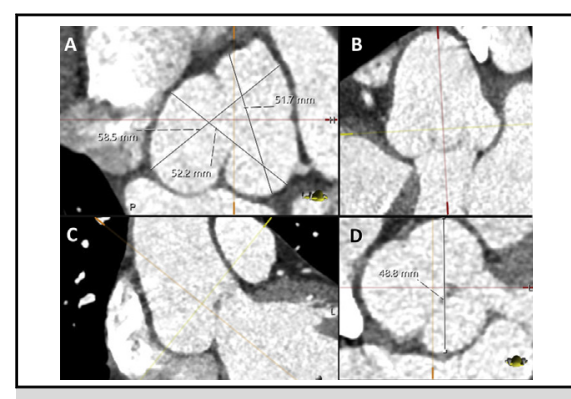

MPR images $(A, B, C)$ provide more accurate information regarding aortic root anatomy than simple transverse (D), coronar, or sagittal images alone.

\section{Central Message}

Decision making in aortic surgery is of paramount importance. Although algorithms may be important tools, clinical judgment has an important role to select the right patients for surgery.

See Article page 1733 . group, $17 \%$ of patients had aortic dissection or rupture within a mean follow-up time of 3 years compared with $3 \%$ in the group in whom surgical treatment was not considered.

Unfortunately, there are some drawbacks of this seemingly simple approach regarding methods used and patient-related factors:

- The authors used standard axial imaging to measure the diameter of the aorta, although multiplanar reformatting has been shown to be more accurate in identifying the true diameter and is the method of choice in the current guidelines. ${ }^{3}$ This has certainly introduced a bias, especially in the group with an aortic diameter of 4 to $5 \mathrm{~cm}$.

- The range of 4 to $5 \mathrm{~cm}$ is quite large. Recommending surgery to patients with a normal functioning bicuspid aortic valve without associated defects at $4 \mathrm{~cm}$ will result in a large number of patients who have not yet been considered surgical candidates. Because the authors' recommendation is largely independent of age and comorbidities, this exposes a group of patients to the risk of surgery in whom the risk of dissection is largely unknown.

- Presence of a connective tissue disorder was considered a major modifying factor and prompted surgery in patients presenting with aortic diameters of 4 to $5 \mathrm{~cm}$. Marfan and 
Loeys-Dietz syndrome are just 2 examples. Over the past decade, hundreds of genes have been implicated in syndromic but also nonsyndromic forms of thoracic aortic disease. The question is, did all patients undergo systematic clinical evaluation and genetic testing to eliminate potential selection bias? The implication would be that from now on all patients with a 4-cm aorta would need to undergo genetic testing before a decision can be made. Furthermore, we have learned that there are marked differences regarding the risk of dissection between different mutations, for example, transforming growth factor- $\beta$ receptor and SMAD3 mutations in patients diagnosed with Loeys-Dietz syndrome. This has not been taken into account in the present study.

- The authors did not differentiate between enlargement of the root and the ascending aorta, although the root is $25 \%$ larger than the ascending aorta in healthy individuals.

- It is unclear whether the patients who refused surgery received the same expert medical treatment than the group of patients who were primarily not considered for surgery. In this study, low mortality was the main outcome parameter for success after aortic surgery. Over the past decade, it has been shown that mortality has become a poor outcome parameter for routine ascending and root surgery. Perioperative neurologic events have become the main factor influencing quality of life after aortic surgery. Therefore, the potential risk of stroke is certainly an important factor when discussing prophylactic surgery in patients with aortic diameters less than $5 \mathrm{~cm}$ and known risk factors for stroke. In the current study, the authors did not differentiate whether patients needed circulatory arrest to remove the entire aneurysmal segment or not.

The most important thing in caring for patients with aortic aneurysms is to prevent dissection and its sequelae.
We strongly advocate early surgery in experienced centers, but decision-making in aortic surgery is a process. It involves meeting the patient in person, reviewing his medical history, and discussing his overall situation. Although the authors stress the adherence to their algorithm, we can assume that after all, clinical judgment still played an important role in selecting patients for surgery.

Although the current study may not answer all our questions, it is certainly an important contribution because it shows that once a surgeon with the necessary knowledge and experience has seen a patient, aortic events are rare.

In a recent editorial in The New England Journal of Medicine, Fried ${ }^{4}$ discusses the fact that "the greater the uncertainty surrounding the options and the greater the clinician's ambivalence about the "right" choice, the greater the likelihood that the patient will be asked to make the decision." Although, prima facie, this seems quite natural but it is not the right thing to do. We as surgeons have to make an effort in each and every patient to give a clear recommendation. If we can't decide, how can the patient?

\section{References}

1. National Institutes of Health. The William Osler papers. Available at: https:// profiles.nlm.nih.gov/ps/retrieve/Narrative/GF/p-nid/348. Accessed September $20,2018$.

2. Saeyeldin A, Zafar MA, Li Y, Tanweer M, Abdelbaky M, Gryaznov A, et al. Decision-making algorithm for ascending aortic aneurysm-effectiveness in clinical application? J Thorac Cardiovasc Surg. 2019;157:1733-45.

3. Erbel R, Aboyans V, Boileau C, Bossone E, Bartolomeo RD, Eggebrecht H, et al ESC Committee for Practice Guidelines. 2014 ESC Guidelines on the diagnosis and treatment of aortic diseases: document covering acute and chronic aortic diseases of the thoracic and abdominal aorta of the adult. Eur Heart J. 2014;35: 2873-926.

4. Fried TR. Shared decision making-finding the sweet spot. N Engl J Med. 2016; 374:104-6. 